\title{
Modelling societal transitions with agent transformation
}

\author{
Michel Schilperoord · Jan Rotmans • \\ Noam Bergman
}

Published online: 23 September 2008

(C) The Author(s) 2008. This article is published with open access at Springerlink.com

\begin{abstract}
Transition models explain long-term and large-scale processes fundamentally changing the structure of a societal system. Our concern is that most transition models are too static. Although they capture a move of focus from static equilibria to transitions between dynamic equilibria, they are still rooted in an "equilibriumist" approach. Improvement is possible with agent-based models that give attention to endogenous system processes called "transformation processes". These models can render far more dynamic pictures of societal systems in transition, and are no longer remote from descriptions in the emerging transition literature.
\end{abstract}

Keywords Societal transitions · Integrated sustainability assessment · Agent-based modelling

\section{Introduction: models of societal transitions}

Transition models can be defined as evolutionary models that explain long-term and large-scale processes fundamentally changing the structure of a societal system. This

\footnotetext{
M. Schilperoord $(\varangle) \cdot$ J. Rotmans

Faculty of Social Sciences, Dutch Research Institute for Transitions, Erasmus University Rotterdam, P.O. Box 1738, 3000 DR Rotterdam, The Netherlands

e-mail: schilperoord@fsw.eur.nl

url: http://www.eur.nl/fsw/staff/homepages/schilperoord/

J. Rotmans

e-mail: rotmans@fsw.eur.nl

url: http://www.eur.nl/fsw/staff/homepages/rotmans/

N. Bergman

Environmental Change Institute Dyson, University of Oxford, Perrins Building, South Parks Road, Oxford OX1 3QY, UK

e-mail: noam.bergman@ouce.ox.ac.uk

url: www.eci.ox.ac.uk/people/bergmannoam.php
} 
definition fits with a large and varied body of modelling work done over the past thirty years. The focus of these models may be economic evolution-for example Nelson-Winter style industry models (Nelson and Winter 1982). It may be technological evolution-for example NK models drawing parallels between technological and biological evolution (Kauffman 1989; Frenken 2006). It may be political evolutionfor example multi-agent implementations for evolving political strategies in computer tournaments (Axelrod 1997; Fowler and Laver 2008). It may be social and cultural evolution-for example dealing with structural change in dynamic social networks (Schelling 1971; Watts and Strogatz 1998). Or it may be the co-evolution of human and ecological systems (Holling and Gunderson 2002).

We report on a modelling exercise part of the MATISSE project ${ }^{1}$ implementing a new class of evolutionary models that focus on societal transitions towards sustainability. These models are explorative in the sense that they help modellers and stakeholders to explore possible transition pathways. They are integrated models emphasizing that societal systems are comprised of inter-locking sub-systems and that above mentioned evolutions cannot be neatly separated. They implement different sources and types of uncertainties (van Asselt and Rotmans 2002). And, as we will argue later, they are highly dynamic models.

This work builds on two pillars. The first pillar is the emerging transition science. This young research field is producing studies of technological and societal transitions in their own right, as phenomena as such. The literature in transition studies varies from historical case studies (Geels 2002) to governance models (Rotmans and Loorbach 2008). There is already a theory of technological transitions (Rip and Kemp 1998; Geels 2002) that is currently being integrated into a theory for studying societal transitions (Rotmans 2005).

The other pillar is the science and application of Integrated Sustainability Assessment (ISA). This field seeks to advance the portfolio of models and participatory methods that can support the development of integrated sustainability policies. Much of the work in the MATISSE project is dedicated to developing ISA in EU policy-making (Weaver et al. 2007) with applications in agriculture, mobility, water and housing. Experiences with transition models within MATISSE can provide elements of a future research agenda for ISA models (Rotmans 2006).

At this stage, our ambition is to develop relatively simple prototypes of ISAmodels that are able to generate transition patterns. The prototypes are concentrated on social and cultural change, not (yet) economic change. The purpose of this modelling exercise is to produce building blocks for a new generation of ISA-models and test for relevance for EU policy-making.

\subsection{Most transition models are too static}

This paper coincides with reports on the first wave of transition models describing aspects of transitions to a hydrogen-based road transport system. Aspects covered

\footnotetext{
${ }^{1}$ Methods and Tools for Integrated Sustainability Assessment (MATISSE). www.matisse-project.net.
} 
include the competition of technological platforms (Struben and Sterman 2006), the roles for government and investors (Greene et al. 2006), the differences in and between regions (Struben 2006), the sequences of stepwise technological transitions (Schwoon et al. 2006), and the reduction of greenhouse gas emissions (Greene et al. 2006).

At a general level, these reports explore possible transition pathways toward transport systems that are dominated by hydrogen technologies, which involves a much smaller scope than exploring pathways toward sustainable mobility. Because evolutions are collapsed into economics, they are not integrated (yet). And there is no plurality in (stakeholder) perspectives. For these reasons, they are not suitable to support an ISA.

Our main concern with most of these transition models is that they are too static. Although they show a move of focus from static equilibria to transitions between dynamic equilibria, they are still rooted in an "equilibriumist" approach (Laver 2005). In other words, their development and use still follows a standard workflow of tasks, including: identifying the (exogenously determined) key components of the system; describing the interactions between components; specifying the model and solving for equilibrium; comparison of forecast outputs. But from a transition perspective the period in between two equilibria, characterized by chaos and instability, is most interesting, because that forms an important impetus for fundamental change.

In these attempts to approach transitions with equilibriumist models, (dynamic) equilibria appear to change only in response to unanticipated shocks, such as a big marketing effect or a technological breakthrough. A societal system such as a road transport system thus appears to change in unpredictable manner, rather than evolve endogenously. And societal actors are assumed to be unitary actors with fixed (exogenously determined) identities. This is all far remote from transition theory.

Transition literature renders a far more dynamic picture of societal systems. Its first attention goes to endogenous system processes called "transformation processes". It considers a societal system capable of innovating itself, through various mechanisms that bring "fitter" functionings to higher scale levels, and downscale the less fit. These mechanisms may be market-driven, but in general they are a complex mix of economic, political, social, cultural, and ecological mechanisms that cannot be collapsed into economics alone.

At the same time, transition literature is not blind for exogenous processes that influence the direction and speed of system innovation. Recent theoretical work attempts to explain how exactly transformation processes can be conditioned by socalled "landscape" changes. It appears that different evolutionary paths are possible, having different balances and interplays between endogenous transformation processes and exogenous landscape changes (Geels and Schot 2007).

This all poses a big question for modellers: How can transition processes be "best" conceptualized and represented in a computer simulation model? This report continues with a description of what we have come to think are building blocks for transition models with endogenous transformation processes. First results with a prototype implementation are discussed and extensions are suggested. 


\section{Building blocks for transition models}

\subsection{Conceptualizing transitions and transformation processes}

This paper describes the implementation of a new conceptual framework for modelling societal transitions (Haxeltine et al. 2008), using and contributing to the development of a comprehensive transition theory. This framework provides generic concepts that are designed to help modellers to describe the essential features of transition processes.

This framework's core concepts—regime, niches and landscape-are based on an understanding of transitions as possible outcomes of the dynamic interplay of the system's dominant functioning, the "regime", on the highest scale level in the system, and the alternative functionings, the "niches", on lower scale levels. The "landscape" describes exogenous changes outside of the system that can affect the regime and the niches. We speak of a transition when the interplay of the regime, the niches and the landscape modulates and involves fundamental change of the regime.

This framework elaborates the regime and niche concepts in terms of their internal dynamics; i.e., how the regime and the niches generate resources, and how they allocate their resources to build up their structures. The latter is conceptualized as consisting of physical and institutional capacity, both of which are oriented towards supporting a particular set of practices that define a regime or a niche. This idea of metabolism of regime and niches branches out to a broader definition of a regime or a niche in terms of its structures (institutional settings), cultures (prevailing perspectives) and practices (rules, routines and habits), which is suitable for a rich representation using stocks and flows interacting over space-time dimensions.

This description of semi-autonomous processes within the regime and the niches is augmented with descriptions of their continuous interactions with the landscape and the "support canvas", which consists of a large and highly diverse population of societal actors (consumers, citizens, companies, or other). The framework offers the "support mechanism" as a way to capture a close two-way coupling between regimeniches and the actors, which is conditioned by the effects of exogenous landscape changes. The support mechanism casts changes of the regime and niche practices as outcomes of continuous adaptation and learning, that are pivotal in the competition between the regime and niches for the support (political, consumptive, investment, or other) from many different actors with different preferences-much in the same way as changes of policy positions are pivotal in the competition of political parties for the support from voters. This continuous interplay involves learning about practices, both collectively and individually, which is an important element of transitions. Landscape changes indirectly change the regime and niche practices, via the support mechanism, principally by changing the distribution of actor preferences.

The linkages between these two descriptions-one concentrating on the buildup of structures, the other on the buildup of support-are as follows: On the one end, a regime or a niche is considered capable of galvanizing part of its support into new resources to build up its structures. At the other end, its structures, in particular its institutional capacity, help a regime or a niche to find new support for its practices.

Using these concepts and descriptions as a foundation, we now define transitions as structured sequences of "transformations" culminating in a fundamental change 
Table 1 Our implementation of five scale levels

\begin{tabular}{ll}
\hline Landscape (Macro) & Signals \\
Regime (Meso) & Collective agent \\
Empowered niches & Collective agents \\
Niches (Micro) & Collective agents \\
Support canvas (Undercurrent) & Individual agents \\
\hline
\end{tabular}

of a societal system's dominant functioning, the regime. We emphasize that this is a modelling definition, applying our ideas of discretization of transitions, which needs to be distinguished from our understanding of transitions as continuous processes.

Transformations can be defined as processes by which the regime and the alternative functionings on the lower niche levels co-evolve in ways that significantly alter the balance of their respective scale levels and, consequently their interaction patterns. We consider, for example, a transformation when a niche has grown strong enough, principally in terms of its institutional capacity, to become effective competitors to the regime-we call this "niche empowerment". This type of transformation, like most transformations, does not fundamentally change the regime, but they can bring the system closer to or further away from a transition.

For implementation of these ideas, five scale levels are distinguished (Table 1). They are the macro, meso and micro levels corresponding to the landscape, the regime and the niches respectively, defined in transition theory (Geels 2002; Rotmans 2006). Added are the levels of "empowered niches", between micro and meso, and the support canvas below micro, also called "undercurrent". Using these five levels, we believe it is possible to describe the full range of transformative dynamics in transitions. To illustrate this, the story of a "micro-to-meso" transition can be told as a line of transformations: emergence of new niches from the undercurrent, clustering of niches, empowerment of niche clusters, and competition between empowered niches and the regime, culminating in change of the regime - all under the impact of landscape changes.

Transformations, a system's main processes, are entangled with different types of "background" processes such as: the birth and death of niches, clustering of niches, attacks of empowered niches to the regime, and absorption of niches by the regime. Interactions like these are, in many cases, about the access to and allocation of resources, and are mediated by a system's structures, cultures, and practices. Details are reported in Haxeltine et al. (2008).

\subsection{Agent-based representation of transition and transformation processes}

The regime, empowered niches and niches are implemented as "collective agents" (Table 1, right column). They are constellations of agents sharing a functioning, and therefore sharing certain structures, cultures, and practices. The undercurrent is comprised of individual agents that support the functionings of collective agents. The landscape exists outside the system and is represented as exogenous signals.

The dynamic behaviour of these agents is simulated using a combination of agentbased techniques and system dynamics modelling.

The agent-based model portrays the competition of collective agents for the support from the individual agents (consumers, citizens, other actors). This model puts 
emphasis on how collective agents search for information and make their decisions on how to adapt their functioning to increase their support. We assume that the collective agents are not strategically forward looking but adaptively learning; i.e. their decisions and interactions on cycle $c+1$ are conditioned on the system history up to cycle $c$ (Laver 2005). The collective agents are not capable of anticipatory behaviours.

The system dynamics model, which is linked to this agent-based model, portrays how the collective agents allocate their resources, obtained from their support, to build up their institutional and physical capacities. We assume that this is semiautonomous behaviour that can be captured using a set of system dynamics equations.

The agent-based model also simulates in some detail the decision-making process of individual agents, about which collective agent they decide to support. We assume an underlying process of individual and social learning about the perceived attractiveness of the functionings of collective agents, which is fed by information on how closely these functionings match their own preferences for consumption patterns and/or lifestyles. The support canvas, implemented as a multi-dimensional "practice space", provides the underlying spatial characterization of consumer preferences, in the tradition of the agent-based models of Kollman et al. $(1992,1998)$ and extensions thereof (Laver 2005; Laver and Schilperoord 2007).

Exogenous landscape signals can significantly impact the dynamics in this system. We assume that these signals have an important impact on the preferences of individual agents. This generates complex and partially unpredictable patterns of movements in the practice space of collective and individual agents.

The two-way coupling in the model between the behaviours of collective agents and individual agents, in the form of the support mechanism, means that those movements can produce sudden, non-linear effects. Based on this fact, we find it intriguing to use the simulation model for experiments about the stabilizing and/or destabilizing effects of information about the scales of functioning, such as the levels of support and/or the institutional capacity of the collective agents.

This line of experimentation led us to the idea of "agent transformation", which is the phenomenon that we have primarily focussed on in our simulation work. Conceptually, we make the distinction that the regime, the empowered niches and the niches are truly different kinds of agents, having different roles in transitions-i.e., they are more than just system functionings on different scale levels. We implemented this idea using different types of agents - regime agents, empowered niche agents, and niche agents - that deploy different behavioural rules with regard to their decisionmaking and adaptive learning. In addition, we defined two sets of criteria for transformations of these types of agents, looking at their levels of support and their institutional capacity. If the first set of (weaker) criteria is met, a niche agent transform into an empowered niche agent. If the second (stronger) set of criteria is met, the latter will transform into a regime agent. These two sets of criteria also define the reverse transformations-from regime to empowered niche, and from empowered niche to niche. 


\section{Transition models with agent transformation}

\subsection{The model}

A large population of (10000 or more) agents is created representing a heterogeneous world of consumers. The consumer agents are endowed with ideal points that express their preferred consumption pattern. In addition, a small population of (no more than 10) collective agents is created that are dynamically composed out of the consumer agents. The collective agents are representative of distinct functionings of the societal system, i.e. the regime and the niches, and comprise coherent combinations of structures, cultures, and practices. They are most akin to institutional agents.

The ideal points of consumer agents and the functionings of collective agents are encoded as coordinates within a multi-dimensional "practice space". The dimensions of the practice space are analyst-specified, typically ranging in number between two and six practices. Each dimension (axis) spans a continuous range from -100 to 100 , with both end points associated with particular analyst-specified practices and corresponding consumption patterns and/or lifestyles.

At initialization, the ideal points of the consumer agents are randomly drawn from a multi-modal Gaussian distribution, with means and variances according to analystspecified consumer groups (traditional mainstream, environmentally conscious). The initial number of collective agents and their functionings are set to analyst-specified values.

Following a cold start, the system evolves:

(i) Each consumer agent is dynamically connected to one collective agent. It is said to "support" that collective agent.

(ii) Collective agents respond to the support decisions of the consumer agents by adapting their functionings.

This two-way process iterates an analyst-specified number of cycles, representative of a time span of 25 to 50 years.

Each cycle, all consumers decide which collective agent they support. Following their decision, they immediately adopt the practices of the collective agent they support. Consumers make their support decision based on individually assessed scores for attractiveness $A$ of all collective agents. They update their scores and select the most attractive agent.

The general form of the attractiveness function is

$$
A_{i}=\alpha s_{i}-D_{i}^{2}+\varepsilon
$$

A collective agent's strength $s$ is calculated as the number of individual agents that support it $-s$ is thus a measure of its scale of functioning. It is assumed that it is attractive for a consumer to adopt practices that are adopted on a large scale $(\alpha>0)$.

The distance $D$ between a consumer and a collective agent is calculated as the Euclidian distance between the consumer's ideal points and the collective agent's functioning; i.e. the distance between their coordinates in the practice space. It is assumed that it is attractive for a consumer to adopt practices that come close to its 
preferred consumption pattern or lifestyle-hence, the minus sign. A random term $\varepsilon$ is added to the equation.

Collective agents can adapt their functioning using one of a number of adaptive rules, which are referred to as "strategies". These strategies spring from different assumptions on the collective agent's adaptive learning and ramifications for how the collective agent will be coupled to its consumer support base (center, off-center) and how it will explore possibilities to extend its support. Commonly used strategies ${ }^{2}$ are:

- STICKER: never change functioning

- AGGREGATOR: on each dimension move toward the mean ideal points of all the individual agents that support it

- HUNTER: if the last functioning change increased support, make the same move; else, reverse heading and move in a heading chosen randomly from the arc $\pm 90^{\circ}$ from the direction now being faced

- PREDATOR: identify the regime; if this is you, stand still; else, make a move towards the regime

Each of these strategies have a satisficing variant; for example

- AGGREGATOR[s]: do not change functioning if support is high enough, else aggregate

It should be noted that, in each strategy, decisions on cycle $c+1$ are conditioned on the system history up to cycle $c$.

The strongest collective agent is referred to as the dominant functioning or the "regime agent". The non-dominant collective agents are referred to as the alternative system functionings or the "niche agents". It is possible that a system evolves niche agents that are strong enough to compete with the regime; these agents are referred to as "empowered niche agents". A system may be dominated by one regime agent, or by a combination of empowered niche agents. In the latter case we speak of a "niche-regime".

The default strategy for the regime agent is aggregator, the empowered niches are predators and the niches are hunters. The use of these strategies is underpinned by the assumption that a regime is orientated at maintaining the status quo, whereas niches are driven by opportunities for change and growth.

A crucial assumption is that if a niche agent continues to grow, at some point it will behave differently, i.e. it will transform into a different type of agent. We implemented the rule that transformation occurs when the scale of functioning of a collective agent, measured by its support, crosses a threshold. A lower threshold, fixed at $15 \%$ of total support, is implemented for transformation of a niche to an empowered niche, or reverse. A higher threshold, fixed at 50\% support, controls the transformation of an empowered niche to a new regime, or reverse. Alternatively, the modeller can use the institutional capacity of a collective agent (calculated as a percentage) as a measure of agent strength, and compare this percentage with these thresholds.

Our first step toward endogenizing transformation processes, using simple thresholds, has the important consequence that a system's composition out of a regime and a

\footnotetext{
${ }^{2} \mathrm{~A}$ larger list of strategies is available in (Fowler and Laver 2008).
} 
number of (empowered) niches is not fixed. This also means that the mix of strategies in a system is not fixed. To endogenize more, we also designed (simple) algorithms for endogenizing background processes such as the birth and death of niches and the clustering of niches; space constraints mean future papers will report these. As a result of all this, the number, functionings, scales and strategies of the collective agents in the system are not fixed but outcomes of the simulation.

This generates complex dynamics of the system that are also subject to impacts from "landscape signals", semi-autonomous changes that are exogenous to the agents. A small set of (typically between two and six) analyst-specified signals are used to setup the main scenarios that need to be considered, including key policies. All signals are time series converted to fit within the range from 0 to 1 , making it easier to concentrate on their differences in shape and timing.

The signals can change the ideal points of consumers, thus reflecting changes in their preferred consumption patterns and/or lifestyles. The ensuing consumer movement in the practice space is controlled by a consumer's "propensity to move" $\lambda_{\text {move }}$. This propensity to move is not the same for every consumer, but proportional to the total pressure $\pi$ that a particular consumer experiences from the signals, and also dependent on the density $d$ of other consumers in its region of the practice space:

$$
\lambda_{\text {move }}=\pi(1-d)^{2}
$$

Pressure and the direction and speed of the ensuing consumer movement are all made dependent on a consumer's ideal points. These parameters are calculated according to a "pressure field" function that returns for a given set of ideal points a vector that is the sum of pressures coming from all signals.

\subsection{Running a simulation}

The simulation model is setup to examine the possibilities for radical change taking the form of "regime change"; i.e. sequences of transformations, or "transition pathways", that culminate in the replacement of the regime agent by an empowered niche agents. The conditions for regime change are set into place by landscape signals; however, landscape signals by themselves are necessary but not sufficient for transformations and regime change, since conditions for transformations are partly evolved by the system itself.

There are different ways of using this model. In our MATISSE case studies on three EU sectors (road transport, Germany; housing, UK; and water, Spain) we have used the model for the discovery of transition pathways over the years 2000 to 2050 . With these models we were able to produce interesting and plausible stories that complement our descriptions of narrative scenarios. We have also used the model to reproduce historically observed data about a well-studied transition (a calibration study on the transition from sailing ships to steam ships in oceanic transport, in the 19th century). In each of these cases we took the main inputs from our narrative scenarios. Those inputs consist of, first of all, the dimensions of the practice space, and based on these dimensions, definitions of the initial state of the system (the initial regime and niches, consumer groups), the scenario for the landscape signals, and the model parameters such as the attractiveness parameter $\alpha$. 
We anticipated the need to set out suites of future simulation experiments for more rigorous analysis of transitions, involving extensive parameter sweeping, computation of "bandwidths" for different types of transition pathways, and identification of transition patterns based on structured sequences of transformations. Our model is already shown to be flexible enough to reproduce different types of pathways (Bergman et al. 2008), each capturing very different patterns of interaction between landscape, regime and niches (Geels and Schot 2007; de Haan 2007). It is also capable of running large batches of simulation runs for producing the bandwidths for pathways, in order to shed light on the uncertainties that originate from non-linearities in agent behaviors.

\section{Analyzing agent transformation in societal transitions}

This is a complex model that will take time to explore systematically. Presented below are initial results and evaluations.

\subsection{Analysis 1 . Scales and evolution of the system}

Before doing any explorative type of analysis, it is necessary to understand better how interactions of (agents on) different scale levels shape the system's evolution. There are principally two ways of how scale levels reenter the simulation's recursive steps. First, the consumer agents use scales in their calculation of attractiveness of the collective agents. Second, historical changes in scale determine the outcome of the strategies for adaptive learning of collective agents, and via agent transformations, their choice of strategy. These feedbacks are analyzed in some detail.

Consider a minimal setup with one regime, four niches, and 10000 consumers with their initial ideal points drawn from a uni-modal Gaussian distribution. We specify the initial functioning of the regime by placing it right in the center of the distribution of consumers, and placing the niches in the peripheries (see Fig. 1). Running this setup without landscape signals, it turns out that this is a stable configuration. Experimenting with different initial positions for the collective agents, we find that the system evolves into the same shape-the regime in the center, the niches in the peripheries. It appears that this is the most (and arguably the only) stable kind of configuration for this system. We should therefore expect two of such configurations to mark the start and end of a transition.

This stable configuration is also highly dynamic. Simple experiments can illustrate this important fact. If we change the regime's strategy from AGGREGATOR (the default strategy) to HUNTER, the regime will no longer be succeed to stay dominant for long time and will eventually collapse. If in another try, we turn niches into PREDATORS instead of (default) HUNTERS, thus instructing them to head straight for the regime's support base, they all lose support quickly (even if all the niches attack simultaneously!). These two experiments teach us about the dynamics of this system. They highlight the contrast between, on the one hand, the high potential for radical change, and on the other hand, the difficulty for niches to compete with the regime, mainly because the regime is able to protect the status quo through continuous incremental changes. 


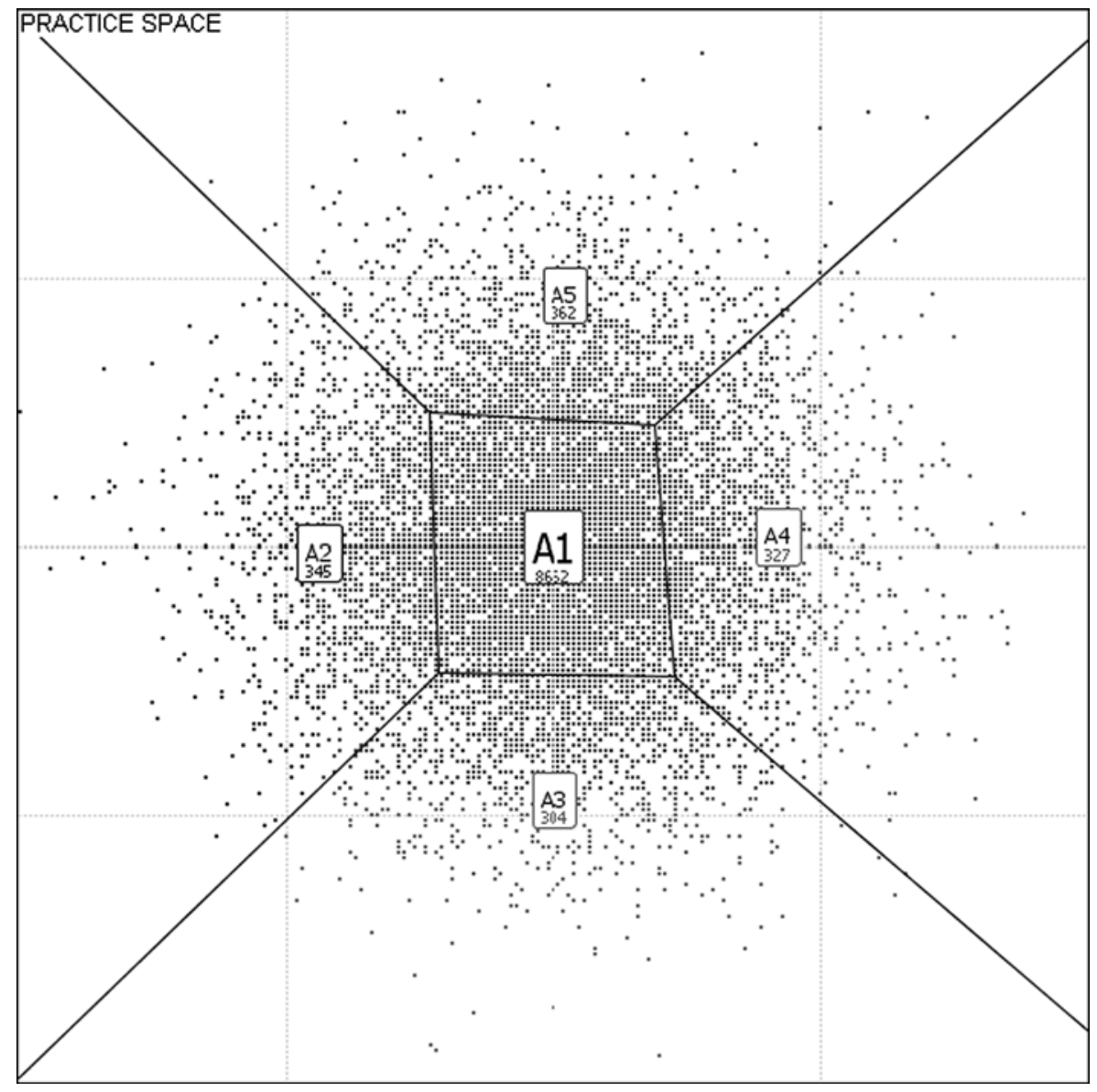

Fig. 1 Dynamic equilibrium of a one-regime, four-niches system. The regime A1 has $87 \%$ of consumer support. The niches cannot move to the centre without losing support. This is a stable, but highly dynamic, system state reached in all our experiments without landscape signals. Notes: A Voronoi tessellation shows what the shape of the regime and the niches would be if $\alpha=0$; i.e. if consumers were to support the nearest collective agent. Voronoi lines allow us to see groups of consumers that support the regime because of its strength, not because of its practices

\subsection{Analysis 2. Transition patterns and pathways}

The second analysis explores in two series of experiments the model's ability to generate transition patterns and pathways. In the first series of experiments no landscape signals are used. In the second series a landscape signal is added.

Here we only provide a summary of the results. A more complete account of the simulations is in Bergman et al. (2008). In the first series of experiments (no landscape) the regime remains stable and there is no transition. The absence of landscape signals means that the ideal points of individual agents are fixed. Given the distribution individual agents' ideal points, niches are unable to gain enough support to reach 


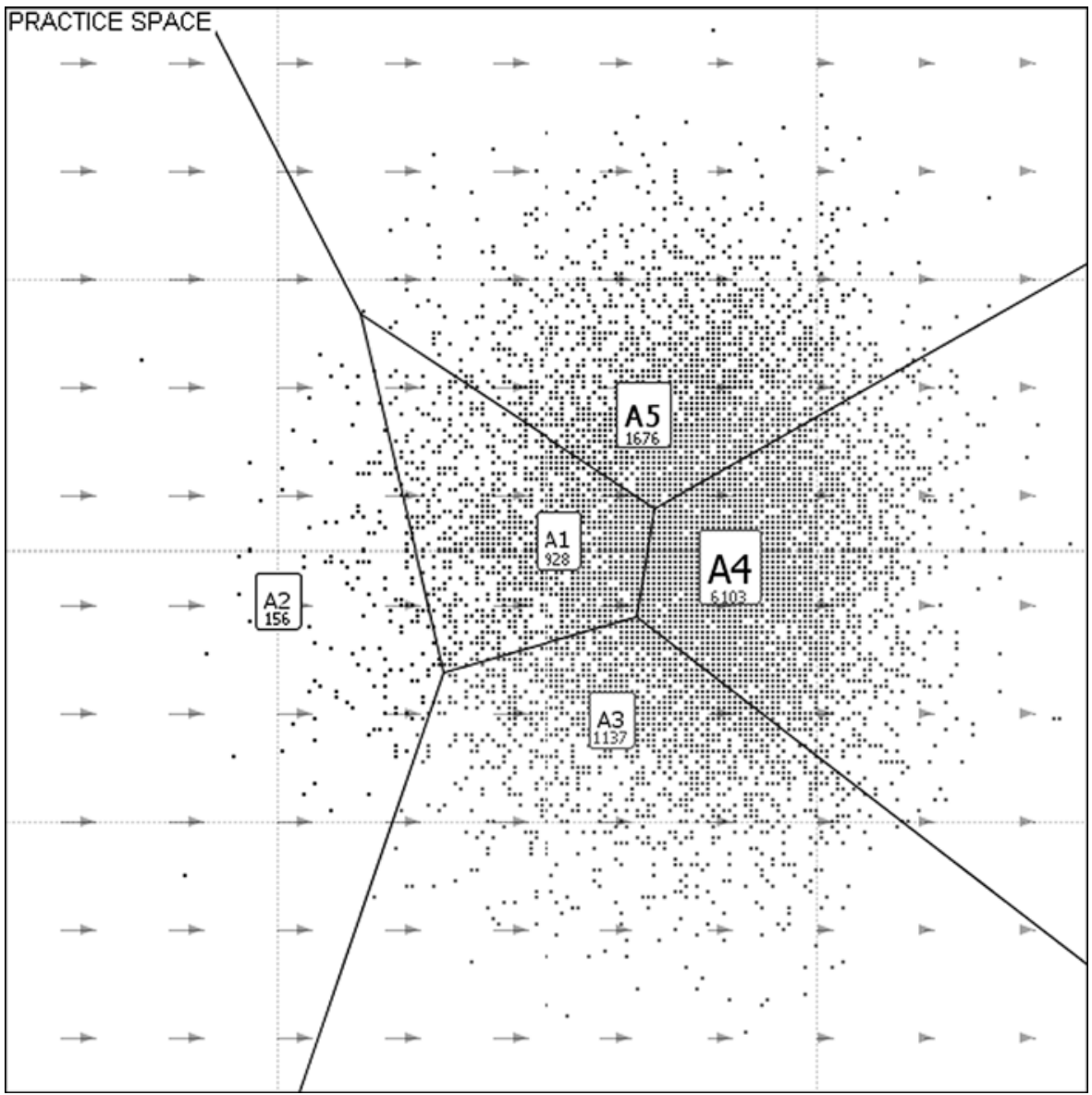

Fig. 2 Towards a new dynamic equilibrium. A landscape signal puts pressure on consumers to change their preferences and the support canvas is moving in the direction of this signal. The regime A1 loses support and eventually niche A4 becomes the new regime. Notes: The pressure of the landscape signal is different depending on position in the practice space. Pressure is assumed to decrease along the direction of the signal

the higher level of empowered niches and to compete with the regime. Each time that the niches pose a threat, the regime is able to adapt quickly enough to thwart it.

The second series of experiments with landscape signal shows an entirely different dynamics (Fig. 2). The landscape signal now has an effect on the ideal points of the individual agents. With landscape signals and a moving support canvas, the problem of achieving a suitable strategy has become much more complicated, for the regime but also for the niches. As a direct consequence, the regime is no longer able to always adapt effectively to niche developments.

Repeated experiments with the same initial state and different landscape signals show the same sequence of four phases in a "micro to meso" transition (Fig. 3). First, the transition begins with existing and new niches gaining support while at the same 

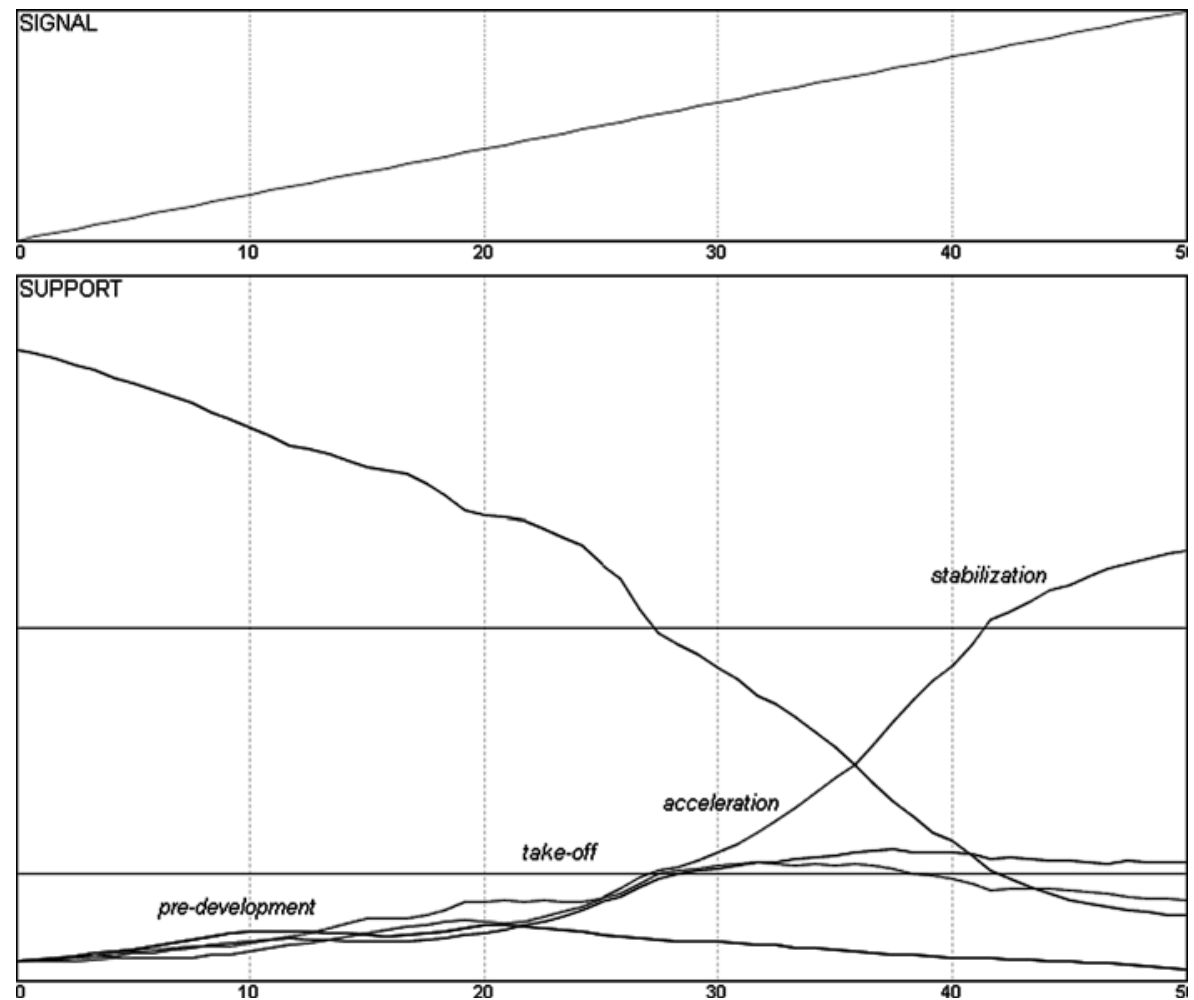

Fig. 3 The four phases in a micro to meso transition: pre-development, take-off, acceleration and stabilization. This chart shows the evolution of the one-regime, four-niche system that is exposed to a gradually intensifying landscape signal, as in Fig. 2. The $X$-axis represents a period of fifty years, which applies to most transitions. The $Y$-axis shows the relative support for the regime and the niches, calculated as the share of consumers that support them. The S-shape curves, characteristic of the multi-level and multi-phase descriptions in transition literature, are a recurring feature in this experiment. Notes: The two transformation thresholds at $15 \%$ and $50 \%$ support are visible. When these thresholds are crossed by a collective agent, that agent transforms into another type of agent. So, when a niche (HUNTER) attracts more than $15 \%$ support it becomes an empowered niche (PREDATOR), and it will become the new regime (AGGREGATOR) if it reaches more than $50 \%$ support

time the regime is losing some of its support. In transition literature this is called the "pre-development" phase.

The growing niches then approach each other, which makes it more likely that they form a combined threat to the regime. This may be the unintended effect of their strategies, or it involves an intended form of clustering to become a new and bigger niche or empowered niche. This is called the "take-off" phase.

At this time the regime but also the empowered niches experience a combination of pressures in which shifts of support go very fast. This is the "acceleration" phase. Experiments show there are three outcomes possible. (i) One empowered niche is able to take over the position of the regime. The regime immediately falls back to a lower scale level. (ii) The regime is able to thwart the combined threat and remains 
dominant. (iii) The regime falls back to the level of empowered niches and competition continues on this level.

After the acceleration phase, the pressures on and within the system have decreased and it enters in a new dynamic equilibrium. This is called the "stabilization" phase. The four phases of pre-development, take-off, acceleration, and stabilization are described in transition literature (Rotmans et al. 2001; Geels 2002).

\subsection{Analysis 3. ISA scenario}

We developed a narrative description of a possible scenario for a transition toward a more sustainable road transport system. As preparation for the model's application to support an actual ISA, we complemented our narrative with simulation results.

Again we only provide a summary of the results. We used six practice dimensions to represent the functionings of the collective agents (the current internal combustion engine regime plus the niches of hybrid, biofuel, and hydrogen technologies, and different transportation modes) and the ideal points of consumer agents (mobility users such as car drivers). The dimensions are emissions performance, price, personal and public modes, ICT use and built environment (Fig. 4).

In addition, we defined a landscape scenario of six signals (exogenous climate change influence on values, higher costs due to increasing oil prices, policies for mobility management, ICT wave, planning of built environment, and public transport investments) that vary in intensity and timing. We assumed that these landscape signals will push car drivers towards preferences for less emissions, more private and public mobility, more use of ICT and more mixed-used zones in the built environment-all this while people are gradually accepting higher prices for their mobility. The time span of the simulations represents the period 2000-2050.

Fig. 4 Impression of the mobility application. We used six practice dimensions to portray the mobility transition. Histograms show the initial distribution of consumer ideal points over the six dimensions. Lines show the initial allocation of support for the regime and the niches. These are all analyst-specified inputs, taken from our description of a narrative scenario for the mobility transition
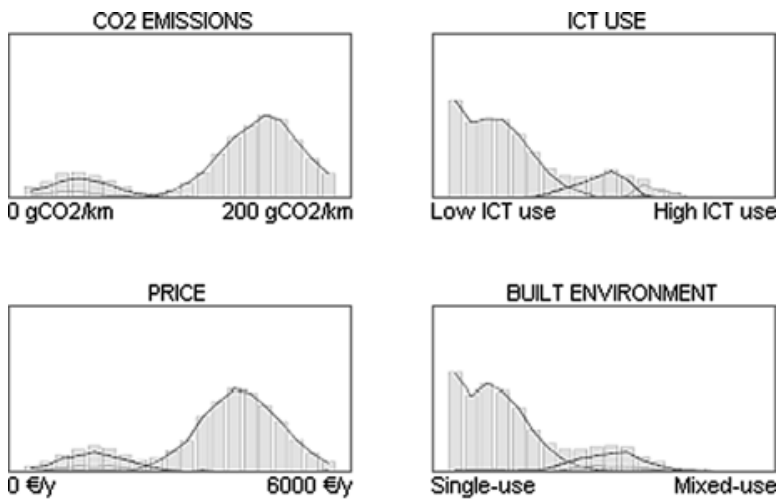

BUILT ENVIRONMENT

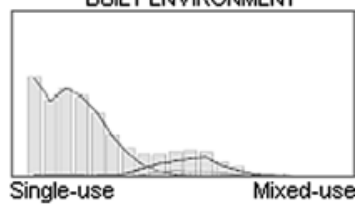

PUBLIC MOBILITY
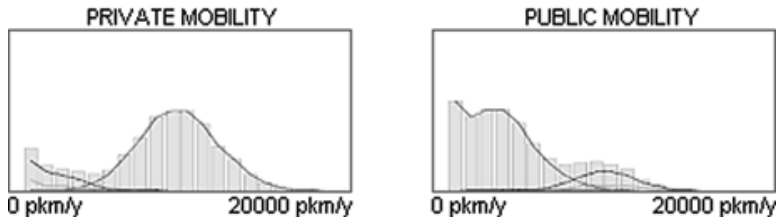
Model parameters were set to their default values. In mobility, the scale of functioning of a collective agent has a considerable impact on decision-making by consumers - think of scale advantages such as a high density of filling stations. The attractiveness parameter $\alpha$ was set to a positive value to capture this effect.

Repeated simulations show two consecutive transitions: 1) from internal combustion engine to hybrids and biofuels; 2) to hydrogen technologies. The first transition is triggered by two landscape signals, climate change effects and oil prices, and can be interpreted as a pre-development for the second transition that involves all six signals and all practice dimensions.

We found that this scenario is remarkably robust against variations of the model parameters, for example against different values for $\alpha$. This result suggests that the simulated transition pathway for mobility shown in Fig. 5 is principally determined by the assumed impact of the landscape signals on the ideal points of consumer agents. We also found that this pathway is not deterministic, and that the dynamic
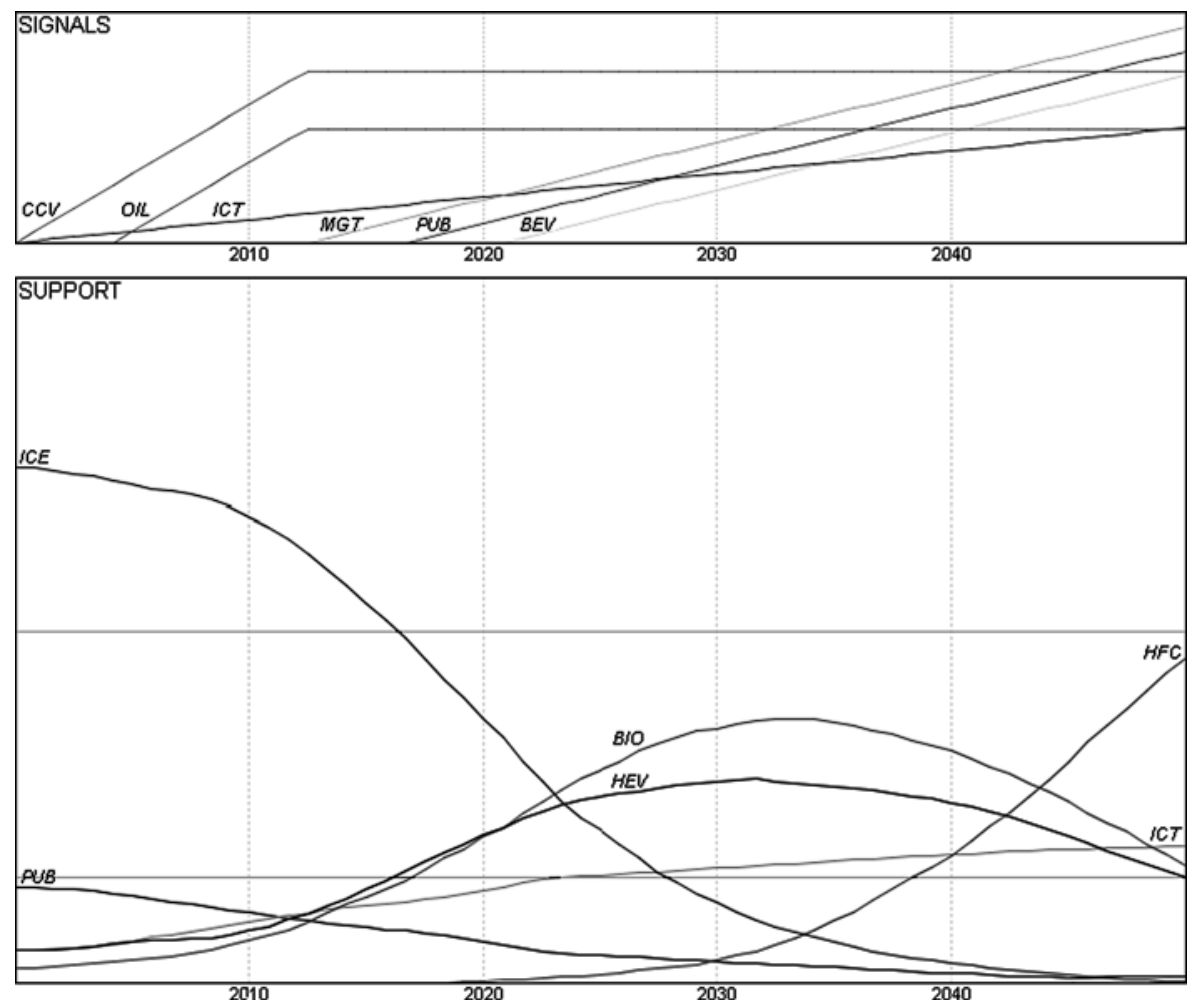

Fig. 5 Base scenario for the mobility transition. The simulation tells a story of two consecutive transitions. First the ICE regime is replaced by a combination of two empowered niches: hybrids and biofuels. Then hybrids and biofuels are replaced by hydrogen cars. Agents: ICE Internal combustion engine, BIO Biofuels, HEV Hybrid electric vehicle, HFC Hydrogen fuel cell, ICT Urban ICT/Low demand, PUB Public transport. Signals: CCV Climate change influence on values, OIL Oil prices/Higher costs, MGT Mobility management, ICT ICT wave, BEV Planning of built environment, PUB Public transport 
interaction of regime, niches and consumers creates realistic bandwidths for this pathway.

\section{Conclusions}

In this paper we set out to develop building blocks for a new generation of (co-) evolutionary models that focus on societal transitions towards sustainability. Over the last three years results have materialized: a new conceptual framework for modelling transitions, new prototypes of transition models, and applications thereof in case studies on EU sectors (transport, housing, water) in the spirit of ISA. This paper distils lessons regarding the basic principles and building blocks for modelling transitions.

First, we set out the development of a generic, agent-based, transition model implementing the new conceptual framework for modelling societal transitions. The most striking findings with this model, reported above, concern the fundamentally different problems of adaptive learning faced by the regime and the niches. In our simulation experiments, we let niches deploy a simple but effective (HUNTER) strategy for their adaptive learning, allowing them to transform themselves into viable alternatives to the regime. The regime deploys a different (AGGREGATOR) strategy to defend itself, which is also simple and effective, but not as robust. We show that with strong signals from the landscape (exogenous changes) certain niches will gain competitive advantage over the regime, which generally means improved conditions for a transition. Our model thus provides a rationale and building blocks for agentbased transition models based on the assumption of adaptive learning by the regime and the niches. Whether this is the only or best rationale remains to be investigated, but some level of confidence can be imparted to the fact that the model is able to reproduce the micro-to-meso transition pathway described in transition literature.

Second, we set out focus on transformation processes. Societal transitions are not solely about the competition of dominant and alternative functionings, but also about system innovation. In simulation results not reported above, we obtained intriguing results with a simple threshold-based rule for agent transformation: one or more niches can transform themselves into the new regime, after passing the intermediate stage of empowered niches. Whilst not providing explanation of (agent) transformation processes, our model helps in an intuitive manner to unravel and break down complex transition patterns into structured sequences of transformations and background processes such as the clustering of niches. All this draws attention to the steps, stages, and sequences of events leading to agent transformations, and the building blocks (algorithms) to capture those-some of which we have already developed.

Third, we set out a way in which the model can be used in the context of an ISA. We show how our model can produce plausible stories about pathways toward a more sustainable road transport system. Over the development of this and other case studies, we were able to rapidly identify and integrate the main ingredients of our stories, based on quantitative and qualitative inputs that are easily obtained. From this fact, we can see potential use of the model as a tool for the envisioning phase of an ISA. 


\subsection{Future work}

We have already implemented some extensions to the basic model; these are briefly described below. A system dynamics module has been added to capture the build up of structures such as physical and institutional capacity in a societal system. This module interacts with the model described above, by calculating investments in new capacity for the regime and the niches based on the support for the regime and the niches. It captures the adaptive learning on the part of "investor agents" such as businesses, financial agents, and government programs. We then used the build up of structures, in particular of institutional capacity, as a second measure of the strength of collective agents, besides consumer support. All this provided useful building blocks for the development of more compelling stories of agent transformationsfor example, we observed a niche that has the support but not yet the structures to grow and become empowered, or a regime that has the structures but no longer the support to maintain the status quo.

Future work will extend the focus on agent transformations on all levels, collective agents and individual agents alike. On the collective levels (micro and meso), we will set out the development of a more complete set of criteria for agent transformation, looking both at an agent's support and at its cumulated structures, together with new strategies of adaptive learning that explicitly involve these criteria. So, if a niche has plenty of support but needs more structures to grow, it will decide to galvanize this support to build up more structures.

On the individual level (the support canvas), we will set out to endogenize an agent's attractiveness parameter $\alpha$ an agent's weights with respect to the practice dimensions, attributes that are now set exogenously. This will hopefully reveal meaningful ways to develop different types of individual agents, having different attributes and possibly different modes of adaptive learning.

We also intend to consider the opposite phenomenon of transformation: "congestion", understood as a system state that blocks transformation. This will hopefully allow the monitoring of transformations in advance, by measuring a decreasing level of congestion in the system before the conditions for actual transformations are set into place.

Perhaps the most important conclusion from this work is that societal transitions can be modelled with a combination of agent-based and system dynamics techniques, underpinning an approach that can be closely linked to the theoretical approaches in transition research, and that, at the same time, can be embedded in the context of an ISA or other strategic policy-making processes. The characterization of societal transitions as processes of adaptive learning and agent transformation is innovative, and this work attempts to show that complex transitions can feasibly be modelled using currently available methods, and that the analysis of transitions as demonstrated here can play an important part in advancing transition theory.

Open Access This article is distributed under the terms of the Creative Commons Attribution Noncommercial License which permits any noncommercial use, distribution, and reproduction in any medium, provided the original author(s) and source are credited. 


\section{References}

Axelrod R (1997) The evolution of strategies in the iterated prisoner's dilemma. In: Axelrod R (ed) The complexity of cooperation: agent-based models of competition and collaboration. Princeton University Press, Princeton

Bergman N, Haxeltine A, Whitmarsh L et al (2008) Modelling socio-technical transition patterns and pathways. J Artif Soc Soc Simul 11(3):7

de Haan J (2007) Pillars of change: a theoretical framework for transition models. Paper presented at the symposium 'modelling transitions to sustainability' at the ESEE 2007 conference 'integrating natural and social sciences for sustainability'

Fowler JH, Laver M (2008) A tournament of party decision rules. J Confl Resolut 52(1):68-92

Frenken K (2006) Innovation, evolution and complexity theory. Edward Elgar, Cheltenham and Northampton

Geels FW (2002) Understanding the dynamics of technological transitions: a co-evolutionary and sociotechnical analysis. Centre for studies of Science, Technology and Society, Twente University, Enschede, p 426

Geels FW, Schot JW (2007) Typology of socio-technical transition pathways. Res Policy 36(3):399-417

Greene DL, Leiby P, Tworek E et al (2006) Systems analysis of hydrogen transition with HytTrans. In: Transportation research board annual meeting 2006, Paper \#06-2538

Haxeltine A, Whitmarsh L, Bergman N et al (2008) Conceptual framework for transition modelling. Int J Innov Sustain Dev 3(1-2):93-114

Holling CS, Gunderson LH (2002) Resilience and adaptive cycles. In: Gunderson LH, Holling CS (eds) Panarchy: understanding transformations in human and ecological systems. Island Press, Washington, pp 25-62

Kauffman S (1989) Adaptation on rugged fitness landscapes. In: Stein E (ed) Lectures in the science of complexity. Addison-Wesley, Reading

Kollman K, Miller J, Page S (1992) Adaptive parties in spatial elections. Am Polit Sci Rev 86:929-937

Kollman K, Miller J, Page S (1998) Political parties and electoral landscapes. Br J Polit Sci 28:139-158

Laver M (2005) Policy and the dynamics of political competition. Am Polit Sci Rev 99(2):263-281

Laver M, Schilperoord M (2007) Spatial models of political competition with endogenous political parties. Philos Trans R Soc B 362(1485):1711-1721

Nelson RR, Winter SG (1982) An evolutionary theory of economic change. Belknap-Harvard University Press, Cambridge

Rip A, Kemp R (1998) Technological change. In: Rayner S, Malone EL (eds) Human choice and climate change, vol 2. Battelle Press, Columbus, pp 327-399

Rotmans J (2005) Societal innovation: between dream and reality lies complexity. ERIM, Erasmus Research Institute of Management, Rotterdam

Rotmans J (2006) Tools for integrated sustainability assessment: a two-track approach. The Integr Assess J 6(4):35-57

Rotmans J, Loorbach D (2008) Transition management: reflexive governance of societal complexity through searching, learning and experimenting. In: Van den Bergh JCJM, Bruinsma FR (eds) The transition to renewable energy: theory and practice. Edward Elgar, Cheltenham

Rotmans J, Kemp R, van Asselt MBA (2001) More evolution than revolution: transition management in public policy. Foresight 3(1):15-32

Schelling T (1971) Dynamic models of segregation. J Math Sociol 1:143-186

Schwoon M, Alkemade F, Frenken K et al (2006) Flexible transition strategies towards future well-towheel chains: an evolutionary modelling approach. FNU working paper

Struben JR (2006) Identifying challenges for sustained adoption of alternative fuel vehicles and infrastructure. MIT sloan research paper no. 4625-06. Available at SSRN: http://ssrn.com/abstract=927012

Struben JR, Sterman J (2006) Transition challenges for alternative fuel vehicle and transportation systems. MIT sloan research paper no. 4587-06. Available at SSRN: http://ssrn.com/abstract=881800

van Asselt MBA, Rotmans J (2002) Uncertainty in integrated assessment modellig: from positivism to pluralism. Clim Chang 54:75-105

Watts DI, Strogatz SH (1998) Collective dynamics off 'small-world'. Networks 393(6684):440-442

Weaver PM, Rotmans J, Turnpenny J et al. (2007) Methods and tools for integrated sustainability assessment (MATISSE): A new European project. In: George C, Kirkpatrick C (eds) Impact assessment and sustainable development: European practice and experience, chapter 9. Edward Elgar, Cheltenham 
Michel Schilperoord is a senior researcher and modeller at DRIFT in Rotterdam. Trained as an econometrist at the Erasmus University Rotterdam, he has participated in scientific research and consultancy work with a strong accent on innovative modelling, in the Netherlands but often outside his homeland. He completed a PhD on Environmental Management in 2005 at the Erasmus International Offcampus PhD Program on Cleaner Production and Industrial Ecology. His research interests are integrated modelling, agent-based simulation, social networks and the complexity sciences. He created different types of software tools and computer models. He published in the field of complexity and innovation dynamics, political simulation, and transition modelling.

Jan Rotmans is the scientific director of DRIFT. In September 2004 he established the new research institute DRIFT: Dutch Research Institute for Transitions and received a position as full professor in transitions and transition management at the Erasmus University Rotterdam. He is one of the founding fathers of Integrated Assessment and an expert in the field of integrated modelling, scenario analysis, indicator development and uncertainty analysis and management. He created the IMAGE and TARGETS Integrated Assessment models. He has more than 200 publications in the field of climate change and global change modeling, sustainable development, and transitions and system innovations.

Noam Bergman is a senior researcher at the Environmental Change Institute at the University of Oxford. He has a background in environmental science and computer modelling. He completed an MSc in Environmental Sciences from the Hebrew University, Jerusalem, with a thesis in chemical oceanography, and completed a PhD on Biogeochemical Earth System Modelling in 2003 at the University of East Anglia. He has researched socio-technical transitions to sustainability and developing transition models, and currently is engaged in research on microgeneration and renewable energy, aimed at supporting sustainability in policy making. 